\title{
Theory, Modeling, and Measurements of Gas Plumes
}

\author{
Gerard P. Jellison*, Herbert J. Mitchell, David P. Miller \\ Spectral Information Technology Applications Center (SITAC), \\ 11781 Lee Jackson Memorial Hwy., Suite 400, Fairfax, VA 22033
}

\begin{abstract}
Spectroscopic measurements of infrared $\mathrm{CO}_{2}$ transitions in gas plumes are reported, and evaluated for their potential to yield a reliable remote sensing technique for determination of plume temperature. Measurements were made on two types of plumes: a sideways-directed plume from a vehicle exhaust, and a stack plume from a propane-burning portable plume generator. Modeling of $\mathrm{CO}_{2}$ emission near $4.25 \mu \mathrm{m}$ from the portable plume generator does not yield a temperature diagnostic due to heavy and unpredictable atmospheric absorption. The $4.25 \mu \mathrm{m}$ band is optically thick in the vehicle exhaust plume measurements. For the vehicle plume, the blackbody Planck equation is used to derive temperatures that agree with results of thermocouple measurements. The ratio of optically thin signals obtained in the vicinity of the $4.25 \mu \mathrm{m}$ and $14.4 \mu \mathrm{m}$ transitions is related to temperature in accordance with Boltzmann statistics. For these experimental conditions, the ratio calculated from the Boltzmann distribution has similar temperature dependence to the ratio obtained from the blackbody Planck equation. Because the ratio of signals obtained at two optically thin wavelengths is independent of concentration, this technique has promise for field measurement of plume temperatures.
\end{abstract}

Keywords: Gas plumes; plume temperature; effluent detection; infrared spectrometry; molecular spectroscopy; hyperspectral; emissive

\section{INTRODUCTION}

Spectroscopic observations of stack plumes can, in principle, provide valuable information about the concentrations and mass flow rates of the effluent gases. Unfortunately, although spectral signatures can provide identification of molecular species, the intensities of spectral features cannot be related to molecular concentrations in any simple way. This problem occurs because infrared emission from molecules is a function of both temperature and concentration. If a reliable spectroscopic technique for temperature determination could be developed, however, these functional dependences could be unraveled, and quantitative concentration measurements might become feasible.

Ongoing research at SITAC is addressing this issue, and this paper gives initial results based on measurements on two types of gas plumes. Results indicate that plume temperature can be derived from the ratio of the measured signal at two infrared $\mathrm{CO}_{2}$ wavelengths. This technique does not require fine spectral resolution, and is insensitive to variations in plume density.

\section{THEORY AND EXISTING TECHNIQUES FOR PLUME TEMPERATURE DETERMINATION}

Molecular energy states are specified by the quantum numbers $v$, which gives a molecule's vibrational energy, and $J$, which gives the angular momentum. Typically, the separation between $v$-states is on the order of tenths of an electron volt $(\mathrm{eV})$; the rotational levels are much closer in energy, on the order of a few hundredths of an $\mathrm{eV}$. The rotationvibration spectral lines observed from polyatomic molecules such as $\mathrm{CO}_{2}$ correspond to transitions in which $v$ changes by \pm 1 , and $J$ changes by $-1,0$, or +1 . Transitions in which the $\Delta J$ between the lower and upper states is equal to $-1,0$, and +1 are referred to as the $P, Q$, and $R$ branches of the spectrum, respectively. Due to constraints on allowed quantum transitions, some molecular bands have no $Q$ branch. $^{1}$

A simulated spectrum of the $4.25 \mu \mathrm{m} \mathrm{CO}_{2}$ transition, with $R$ and $P$ branches indicated, is shown in Figure 1. This figure simulates the signal from an instrument such as the Fourier Transform infrared (FTIR) spectrometer used in SITAC's

* jellisog@sitac.org; phone 1703 591-8546; fax 1703 591-2437 
measurements, which has insufficient spectral resolution to observe the fine line structure caused by the transitions between individual $J$ states. The fine structure is smeared out, but the contours of the $P$ and $R$ branches are resolved.

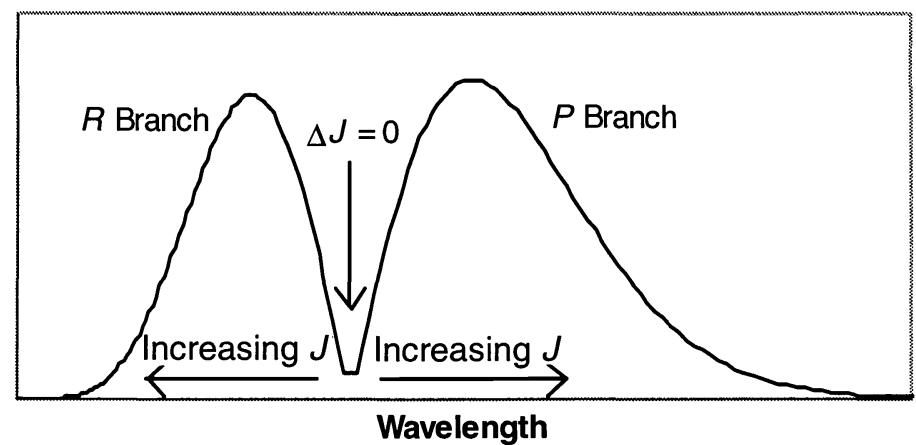

Figure 1. Typical rotation-vibration spectrum.

The $P$ and $R$ branches have maxima corresponding to $J \approx 15$ (for typical plume temperatures). The shapes of the spectra derive from the Boltzmann distribution of molecules over the $J$ states. The number of molecules of energy $E$ is

$$
N_{E}=\frac{N_{o} g_{E}}{Q} \exp \left(-\frac{E}{k T}\right)
$$

where $N_{o}$ is the total number of molecules, $Q$ is the partition function, $g_{E}$ is the degeneracy (the number of quantum states with energy $E$ ), $k$ is Boltzmann's constant, and $T$ is the temperature. As temperature increases, the molecules occupy higher $J$-states (which have higher energies), and the maxima of the $R$ and $P$ branches move farther apart.

Such thermal-induced changes in molecular spectra can, in some cases, be used to measure gas temperature. A 1973 theoretical paper by Chan et al. suggested that the separation between the maxima of the $P$ and $R$ branches of a spectral line could provide a temperature diagnostic for stack gases. ${ }^{2}$ This technique requires excellent spectral resolution, since a $10 \mathrm{~K}$ temperature change in $\mathrm{SO}_{2}$ would change the separation of the maxima by only $0.5 \mathrm{~cm}^{-1}$. Papers by Herget and Brasher $^{3}$ and Herget ${ }^{4}$ describe temperature determination using the intensity ratios of the $P$ and $R$ branches of the 10.4 $\mu \mathrm{m} \mathrm{CO}_{2}$ line. Used on two industrial plumes, the technique gave excellent agreement with the measured in-stack temperatures. Some of this agreement may be fortuitous, however, since the plume temperatures would be expected to be somewhat less than the in-stack temperature. ${ }^{5}$ This technique also requires good spectral resolution. Haus et al. obtained power plant plume temperatures by fitting $\mathrm{CO}_{2}$ line maxima and minima at approximately $4394 \mathrm{~cm}^{-1}$ to line-byline computed values. ${ }^{6}$ Reviewing this work, as well as several other techniques discussed in a review by Gross et al. ${ }^{7}$, leads to the conclusion that most techniques for spectroscopic temperature determination require high spectral resolution.

An additional difficulty is caused by atmospheric absorption. In field measurements on plumes, the "pure" spectrum often will not be observable. The emitted energy passes through some path length of air before it is received by the spectrometer, and if one is observing plume emission from $\mathrm{CO}_{2}$ (for example), the emitted energy can be partially absorbed by the $\mathrm{CO}_{2}$ in the air. This absorption will cause a "hole" around the line center. Only the outer edges of the branches (the so-called red and blue spikes) will be observable. Standard laboratory techniques may not be appropriate to remote measurements of molecular transitions that are subject to significant absorption by the intervening air.

Other published techniques are applicable only in special circumstances. For example, Prengle and collaborators determined plume temperature by measuring the total radiation flux over the spectral range of an FTIR. ${ }^{8,9}$ This method requires good knowledge of the plume emissivity and various geometric factors. Solomon et al. obtained FTIR measurements from an industrial stack. ${ }^{10}$ The spectrum had peaks and dips that could be compared to blackbody spectra. Several temperatures were deduced: the hot plume gas, an absorbing region of cold gas, and the wall (via scattered radiation at high wavenumbers). This technique, unfortunately, requires that the spectrum contain optically thick regions. In recent work, Young extracted plume temperatures from overhead hyperspectral data using a linear relationship between the gas contrast coefficient and the underlying surface radiance. ${ }^{11}$ Using a subregion of the image with constant plume parameters but varying underlying radiance values, a least-squares fit was used to estimate the column density, 
plume radiance, and temperature, with reasonable results. This technique requires a number of pixels with uniform plume parameters, but varying background radiance (clutter).

None of the techniques reviewed are fully relevant to the present study. Based on published research, the need still exists for a reliable temperature determination technique under realistic field conditions.

\section{EXPERIMENTAL ARRANGEMENTS}

\subsection{Plume Sources}

The spectral data to be presented in this section were collected using two plume sources: the exhaust plume from a Ford Excursion $^{\mathrm{TM}}$ engine, and a plume from a portable propane-burning plume generator.

\subsubsection{Vehicle Exhaust Plume}

Measurements were made on an exhaust plume that was generated by a 7.3-liter Ford Excursion ${ }^{\mathrm{TM}}$ operating at controlled fixed idle. To provide a horizontal stack, a 10-cm-diameter aluminum dryer vent hose was attached to the tail pipe. A small aperture 1 meter from the stack exit allowed injection of volatile analytes, using a paint sprayer. This procedure allowed analyte spectra to be measured, and also cooled the plume, providing a range of plume temperatures without changing the spectrometer location. Thermocouples were placed at various positions in the flow to provide temperature data. Both downward-looking (blacktop background) and upward-looking spectrometer data were obtained. The blacktop temperature was determined with a contact probe at various times during the data runs. Data sets were obtained on five occasions from August to October 2002. The plume generation setup is shown in Figure 2.

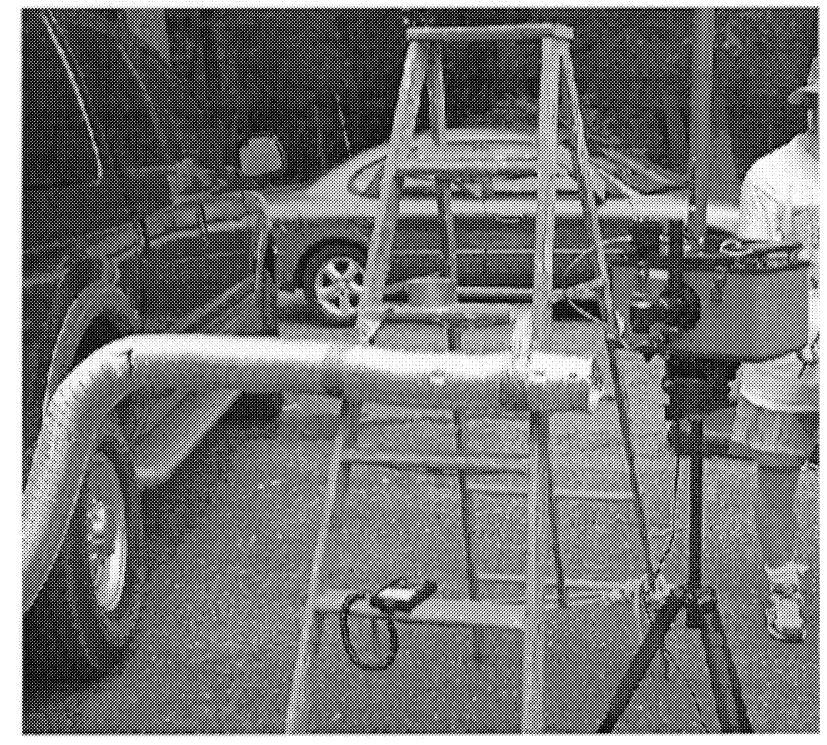

Figure 2. Vehicle exhaust plume setup: horizontal stack with spectroradiometer.

\subsubsection{Portable Plume Generator at Waxahachie}

Spectra from a portable plume generator were collected by SITAC and other organizations on a runway at the Mid-Way Regional Airport near Waxahachie, Texas from October 28 to November 1, 2002. The plume generator was constructed and operated by AeroSurvey, Inc. ${ }^{12}$ Its base unit contains a centrifugal blower that feeds a combustion plenum, in which resides a liquid propane burner. Heated gas from the combustion plenum is fed into an elbow that redirects the gas into a 19-in. diameter vertical stack. The total stack height used for the Waxahachie measurements was approximately 15 feet. Plume temperature was regulated by adjusting the propane delivery pressure to the burner. Target analytes were injected into the plume at controlled flow rates. The SITAC spectrometer observations at Waxahachie were upward-looking, and were taken at positions ranging from 0.25 to $2 \mathrm{~m}$ downwind of the stack. Figure 3 shows the plume generator. 


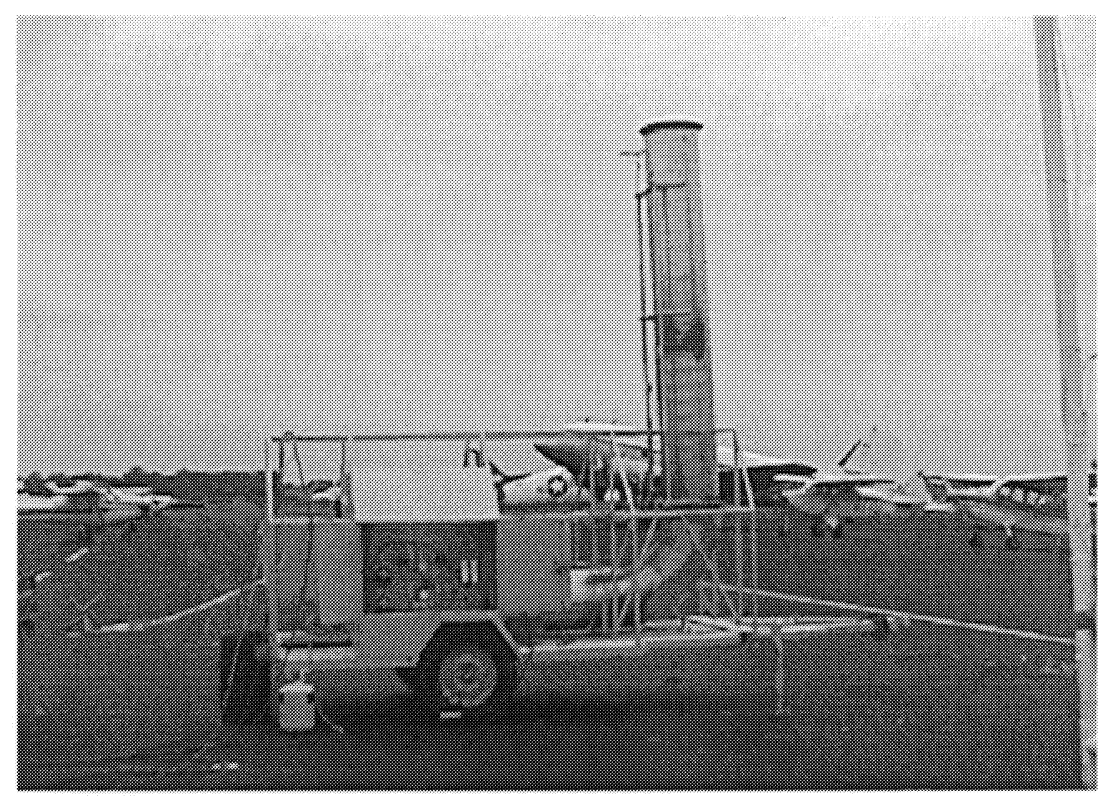

Figure 3. AeroSurvey portable plume generator.

\subsection{Instrumentation}

SITAC's spectral measurements were made using a Designs and Prototypes (D\&P), Inc. MicroFTIR Model 101 spectroradiometer. Useful spectral data were obtained in the range from 3 to $18 \mu \mathrm{m}$. The spectral resolution at $10 \mu \mathrm{m}$ was approximately $40 \mathrm{~nm}$. The single scan time was 16 seconds.

Thermocouple readings were taken from both plumes. Although complete thermocouple results from the Waxahachie collection were not available in time to be incorporated into this analysis, the maximum plume temperature (close to the stack) was noted to be about $500 \mathrm{~K}$. This information is used in the analysis of Section 4.2.3.

\section{MEASUREMENT RESULTS AND ANALYSIS}

A separate publication discusses the detection of analyte species in the plumes at various concentrations and against various backgrounds. ${ }^{13}$ Therefore, this paper is restricted to results relevant to temperature determination. The discussion concentrates on data from $\mathrm{CO}_{2}$ emission lines, since $\mathrm{CO}_{2}$ is ubiquitous in the plumes and the percentage concentration of $\mathrm{CO}_{2}$ should be relatively constant, allowing temperature effects to be isolated and studied.

\subsection{Plume Spectra}

Figure 4 shows spectra from the vehicle exhaust plume at two measured temperatures, $42^{\circ} \mathrm{C}$ and $58^{\circ} \mathrm{C}$, corresponding to different amounts of acetone injection. As expected, the gas spectra appear superimposed on the emission from the background, which for these measurements was blacktop at a measured temperature of $14.8^{\circ} \mathrm{C}$. Of course, atmospheric absorption is not a factor here, since the distance between the vehicle exhaust plume and the spectrometer was negligible. Blackbody curves at the two measured plume temperatures are also shown in the figure. These theoretical curves were normalized by fitting a blackbody spectrum to the $14.8^{\circ} \mathrm{C}$ background signal seen in the data, and then generating new curves at the two desired temperatures. For the most part, the measured spectra do not reach the respective blackbody limits, indicating that the transitions are optically thin. The sole exception occurs in the vicinity of $4.25 \mu \mathrm{m}$; the signals in this wavelength band nearly touch the blackbody curves (the $42^{\circ} \mathrm{C}$ data actually does touch the blackbody curve; the $58^{\circ} \mathrm{C}$ emission is about $80 \%$ of the blackbody limit). Considering the estimated measurement error in the temperatures, this observation is consistent with the conclusion that this is an optically thick transition. It is also worth noting that the hottest, central region of a plume may not be visible at an optically thick wavelength; if the condition for optical thickness is satisfied in the outer regions of a plume, the temperature obtained from a blackbody comparison may not be the maximum temperature along the geometrical line of sight. 




Figure 4. Vehicle exhaust plume spectra with injected acetone at 42 and 58 degrees $C$, compared with blackbody curves.

The $\mathrm{CO}_{2}$ emissions at $4.25 \mu \mathrm{m}\left(2349 \mathrm{~cm}^{-1}\right)$ and $14.9 \mu \mathrm{m}\left(667 \mathrm{~cm}^{-1}\right)$ are shown in detail in Figure 5. The $4.25 \mu \mathrm{m}$ signal is flat-topped. This shape indicates that this transition is optically thick, since this band would otherwise resemble Figure 1 (with $R$ and $P$ branches, but no $Q$ branch). The "wings" of the transition are optically thin. The $14.9 \mu \mathrm{m}$ transition is optically thin and shows $R, P$, and $Q$ branches, as expected from comparison with library spectra.

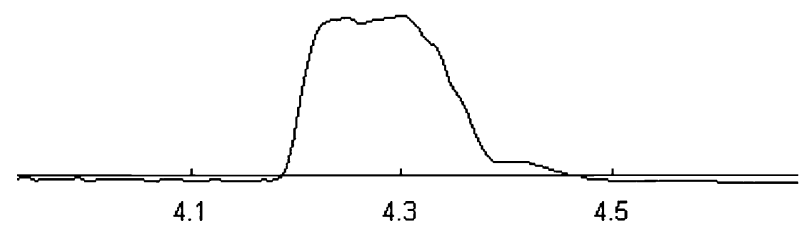

(a) $4.25 \mu \mathrm{m}$ transition

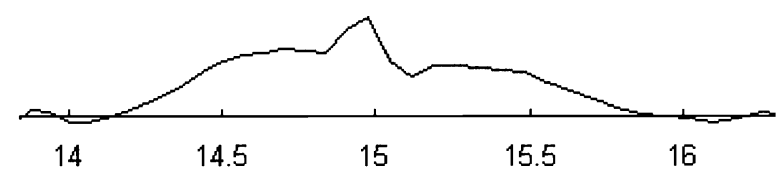

(b) $14.9 \mu \mathrm{m}$ transition

Figure 5. Details of vehicle exhaust plume spectra. The spectra are corrected for the underlying background signal.

Figure 6 shows a typical spectrum from the Waxahachie data; in this case the plume contains no injected analyte. Many of the transitions seen in the vehicle exhaust plume are visible here as well, with some differences in relative intensity. The $4.25 \mu \mathrm{m}$ transition is clearly bifurcated into red and blue spikes, due to atmospheric absorption. The spectrum of this band will be discussed in more detail in the next section.

Comparison with blackbody spectra indicates that none of the transitions in the Waxahachie data appear to be optically thick, although the $4.25 \mu \mathrm{m}$ band may come close. Although the Waxahachie portable plume generator produces a much more impressive plume than does the Ford Excursion ${ }^{\mathrm{TM}}$, the high-intensity (low $J$ value) portion of the $4.25 \mu \mathrm{m}$ band is removed by atmospheric absorption. The red and blue spikes that reach the spectrometer represent the relatively weak wings of the band; hence, it is not surprising that the signal in the Waxahachie data corresponds to optically thin conditions, and does not reach the blackbody limit. 


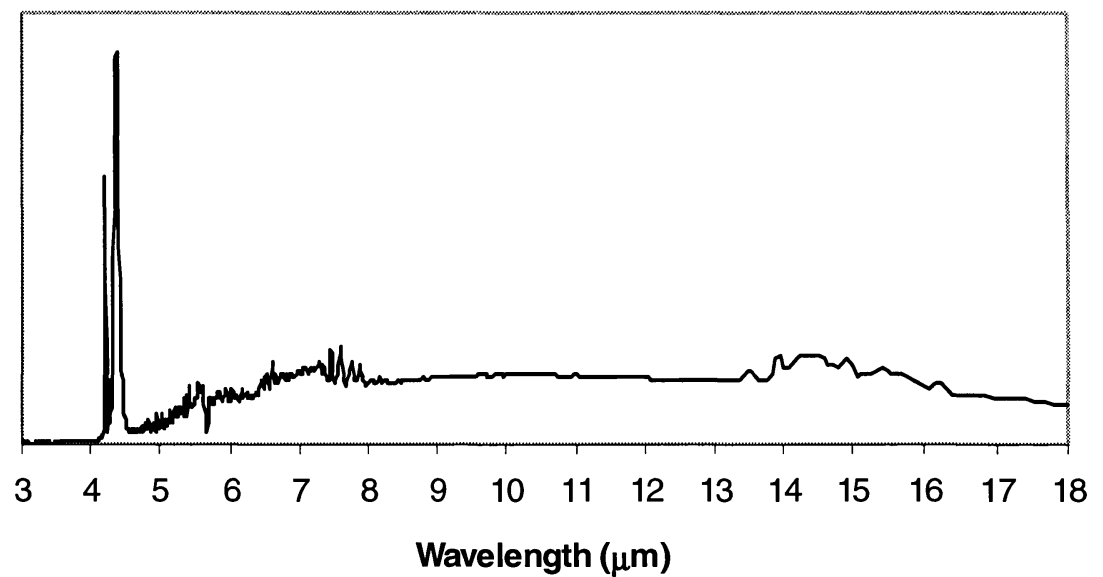

Figure 6. Waxahachie plume spectrum against sky background.

\subsection{Temperature Determination}

\subsubsection{Temperature from Optically Thick Transition}

It has been noted already that an optically thick band is discernable at $4.25 \mu \mathrm{m}$ in the vehicle exhaust plume spectrum. Since the plume is evidently a blackbody at this wavelength, a spectroscopic determination of temperature for these plume data can be obtained from the blackbody Planck equation:

$$
B(T, \lambda)=\frac{2 \pi h c^{2}}{\lambda^{5}} \frac{1}{e^{c h / \lambda k T}-1}
$$

where $B(T, \lambda)$ is the spectral emissive power, $h$ is Planck's constant, and $c$ is the speed of light. Solving for $T$ :

$$
T=\frac{c h / \lambda k}{\ln \left(\frac{2 \pi h c^{2}}{\lambda^{5} N S}+1\right)}
$$

where $S$ is the observed spectrometer signal and $N$ is a normalizing constant. Results are shown in Figure 7 for the data points that are associated with thermocouple temperature measurements. The temperatures determined from Eq. (3) correlate reasonably well with the thermocouple data. ${ }^{*}$

However, one cannot always find an optically thick transition. It would be helpful, therefore, to use spectral profiles or intensities from optically thin transitions to deduce plume temperature. This issue is addressed in the following sections.

\subsubsection{Modeling of $4.25 \mu \mathrm{m}$ Transition}

In the Waxahachie data, as already noted, the $\mathrm{CO}_{2}$ transition at $4.25 \mu \mathrm{m}$ shows a strong absorption "hole" resulting from atmospheric attenuation of the central part of the band. The observed signal consists of the high- $J$ lines that do not experience significant absorption from the $\mathrm{cool} \mathrm{CO}_{2}$ in the air. Since the population of these high rotational states is a strong function of temperature, it might be possible to relate the peak intensity or shape of the red and blue spikes to plume temperature. It might happen, for example, that the atmospheric absorption profile would act as a high-resolution spectral filter; when this filter is applied to the plume emission spectrum, with its steep slope at high $J$ values, a useful variation with temperature might be observed. Hence, some effort was exerted to model the spectrum at $4.25 \mu \mathrm{m}$.

${ }^{*}$ A few data points have intensities at $4.25 \mu \mathrm{m}$ that result in unrealistically high computed temperatures (above $80^{\circ} \mathrm{C}$ ). This may be a calibration error resulting from the radiometric calibration sources used for these measurements, which were considerably cooler than the plume. Another possibility is that the distribution over $\mathrm{CO}_{2}$ vibrational states was not in equilibrium with the translational (thermal) energy of the plume molecules. 


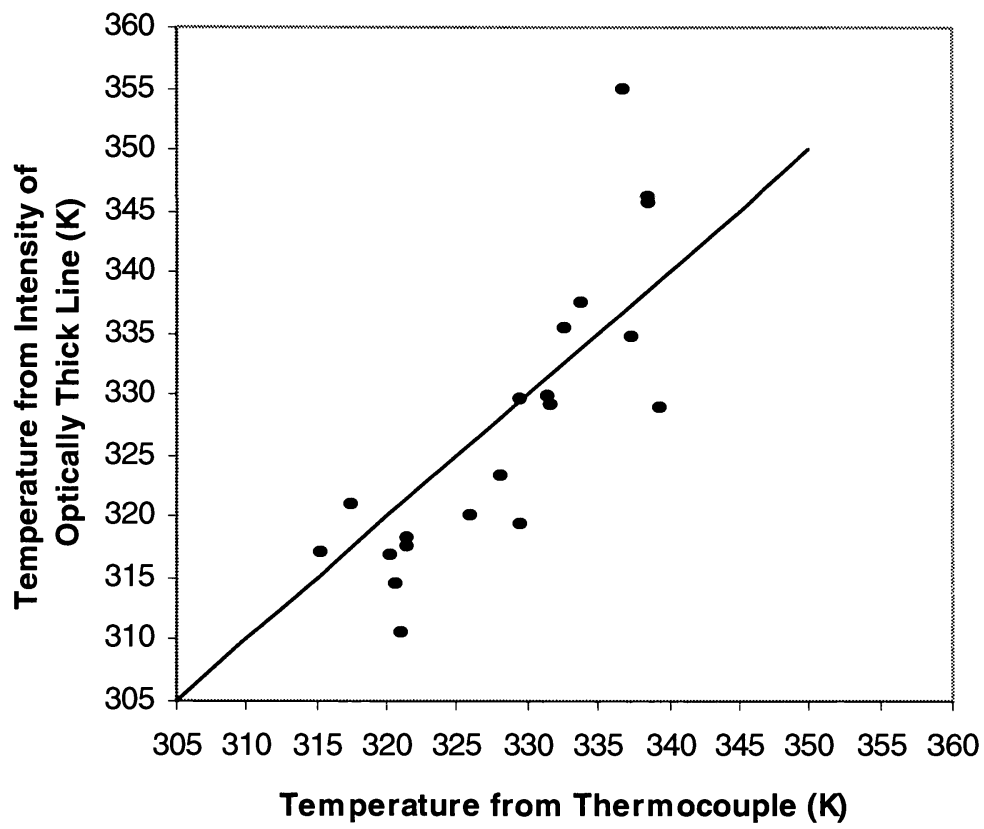

Figure 7. Comparison of spectroscopically-determined temperatures with temperatures from thermocouple measurements (vehicle exhaust plume).

To do this, the wavelengths and intensities of the spectral lines corresponding to various $J$ values were calculated. The wavenumbers of these lines are given by the equation ${ }^{14}$

$$
v=v_{o}+\left(B^{\prime}+B^{\prime \prime}\right) m+\left(B^{\prime}-B^{\prime \prime}\right) m^{2}
$$

where $v_{0}$ is the wavenumber of the line center, $B^{\prime}$ and $B^{\prime \prime}$ are rotational constants for the initial and final states, and $m=-J$ for the $P$ branch and $(J+1)$ for the $R$ branch. The coefficients of the linear and quadratic terms were obtained by measuring the positions of the hyperfine lines in a published spectrum ${ }^{15}$ and obtaining a fit to Eq. (4). The values found were $B^{\prime}+B^{\prime \prime}=0.782 \mathrm{~cm}^{-1}$ and $\left(B^{\prime}-B^{\prime \prime}\right)=-0.0031 \mathrm{~cm}^{-1}$. The intensities were calculated from the equations:

$$
I_{a b s}=C_{a b s}\left(J^{\prime}+J^{\prime \prime}+1\right) \exp \left[-B^{\prime \prime} J^{\prime \prime}\left(J^{\prime \prime}+1\right) h c / k T\right] \text { (for absorption) }
$$

and

$$
I_{e m}=C_{e m}\left(J^{\prime}+J^{\prime \prime}+1\right) \exp \left[-B^{\prime} J^{\prime}\left(J^{\prime}+1\right) h c / k T\right] \text { (for emission) }
$$

where $C_{a b s}$ and $C_{e m}$ are constants (for a given rotation-vibration band). ${ }^{16}$

The resulting simulated spectra showed separable dependences on assumed values for plume temperature and atmospheric $\mathrm{CO}_{2}$ density. For example, the wavelength of peak signal was sensitive to the amount of atmospheric absorption, but not to temperature. Furthermore, the small signal near line center showed almost no temperature dependence. However, the amount of energy received from transitions with large $J$ values did depend strongly on temperature. This dependence suggested that the separation between the peaks of the red and blue spikes might be used to infer atmospheric absorption parameters, and that analysis of the large- $J$ portion of the spectrum might provide a temperature diagnostic. The temperature obtained would not be spatially resolved, of course, but would be a weighted average (by density and temperature) along the optical line of sight.

Unfortunately, these theoretical indications of a temperature diagnostic are not borne out by the currently-available data. Figure 8 shows simulated and observed Waxahachie plume spectra in the vicinity of $4.25 \mu$ m; comparison with Figure 1 shows the effect of atmospheric absorption. Clearly, the correspondence between theory and experiment is not good 
enough to deduce plume temperature by matching data to the model. The observed red and blue spikes are much broader than the simulation, possibly due to the range of temperatures present in the line of sight through the plume. Since absorption by heated $\mathrm{CO}_{2}$ in the outer regions of the plume is probably significant, both spectral profiles involved in computing Figure 8, emission and absorption, are likely to be broadened. Inspection of the data reveals that spectra known to correspond to differing temperatures do not show differences in shape that would allow these temperature differences to be deduced. At this point in the investigation, it appears that spectral observations of a single molecular transition are not likely to yield a workable temperature diagnostic.

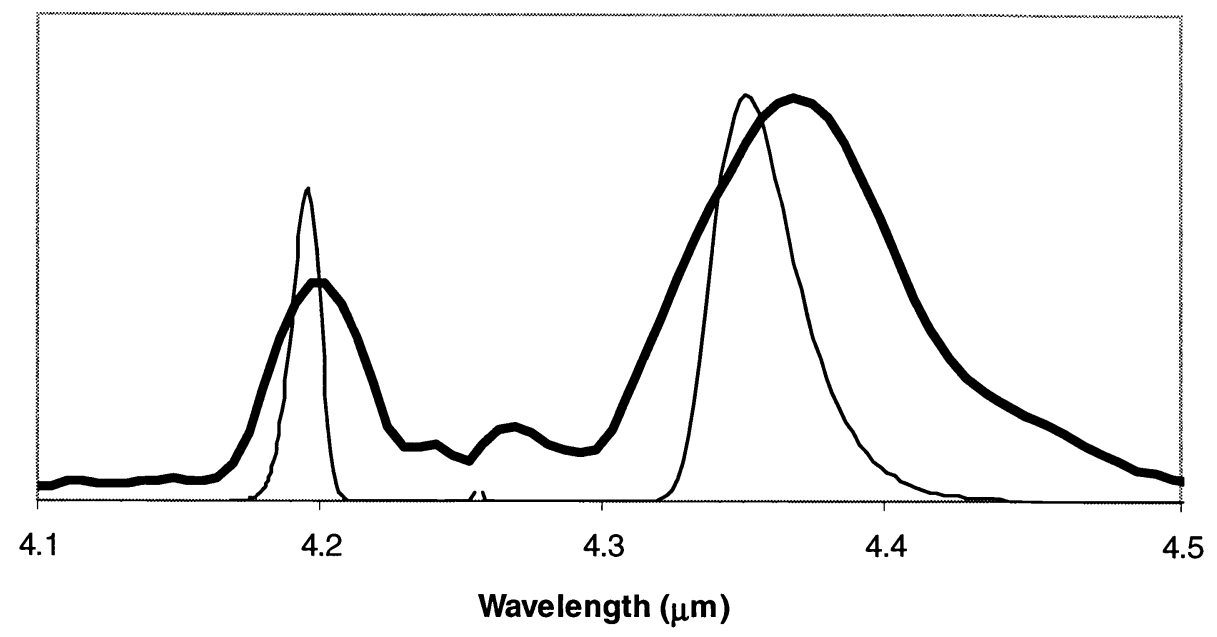

Figure 8. Waxahachie data; simulated (light line) and observed (heavy line) spectra near $4.25 \mu \mathrm{m}$.

\subsubsection{Temperature from Line Ratios}

Another possible temperature diagnostic - the ratio of measured intensity at two widely separated wavelengths - was investigated, and gave more promising results. Intensity at short wavelengths should increase more rapidly with temperature than intensity at long wavelengths; hence the ratio should be temperature-dependent. For optically thick lines, this ratio can be calculated from blackbody theory (Eq. (2)). For optically thin lines, the signal ratio should be related to the Boltzmann distribution (obtained by writing Eq. (1) in terms of vibrational energy levels).

A theoretical curve derived from the blackbody equation, showing the expected intensity at $14.4 \mu \mathrm{m}$ vs. that at $4.4 \mu \mathrm{m}$, is shown in Figure 9. As expected, high temperatures cause a proportionately greater increase in the shorter-wavelength signal. The figure also shows the temperatures corresponding to various points on the curve. For comparison, a curve generated from the Boltzmann distribution (with the partition function assumed to have no significant temperature dependence) is also shown in the figure. There is little difference between the two curves. This agreement occurs because the temperature-dependent terms $\left(\exp (-h c / \lambda k T)\right.$ for the Boltzmann case, and $[\exp (h c / \lambda k T)-1]^{-1}$ for the blackbody case) are nearly equivalent when $\exp (h c / \lambda k T)>>1$, as in these experiments. Hence, even for optically thin lines, the blackbody equation should give intensity ratios that agree with the data.

This prediction was tested with the Waxahachie data, using the ratio of the intensities at $4.40462 \mu \mathrm{m}$ and $14.4371 \mu \mathrm{m}$. These wavelengths were chosen because they had relatively high intensities that did not appear subject to appreciable amounts of absorption. The background contribution to the signal was removed by subtraction, using no-plume observations made at various times during the data collection.

Waxahachie results are shown in Figure 10, along with a scaled version of the theoretical blackbody prediction. The data were taken at four distances from the stack: $0.25,0.5,1$, and $2 \mathrm{~m}$. Since these transitions are optically thin, the absolute values of the intensities do not correspond to blackbody values, as can be seen from Figure 6. Only the ratios at the two wavelengths follow blackbody predictions. Therefore, the blackbody curve has been scaled ( $\mathrm{x}$ and y components multiplied by appropriate constants) to fit the data. The $500 \mathrm{~K}$ point of the theoretical curve has been matched to the rightmost data point, since this corresponds to the highest temperature recorded by the Waxahachie thermocouple array. 


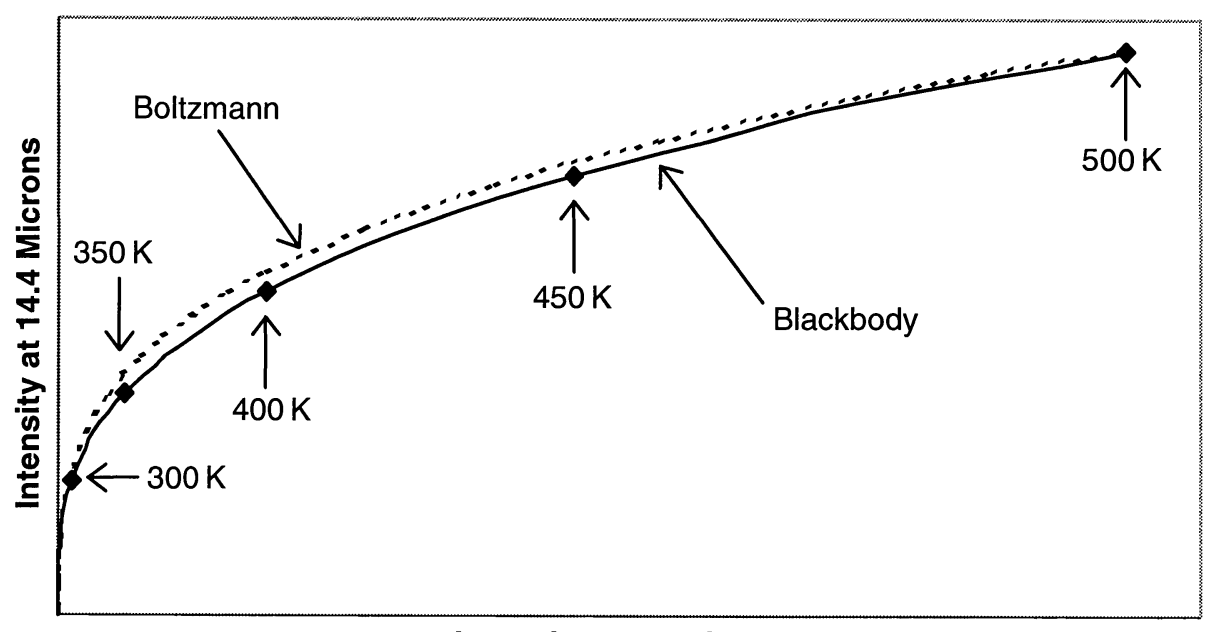

Intensity at 4.4 Microns

Figure 9. Signal at $14.4 \mu \mathrm{m}$ vs. signal at $4.4 \mu \mathrm{m}$ (theoretical). The two curves are scaled to agree at 0 and $500 \mathrm{~K}$.

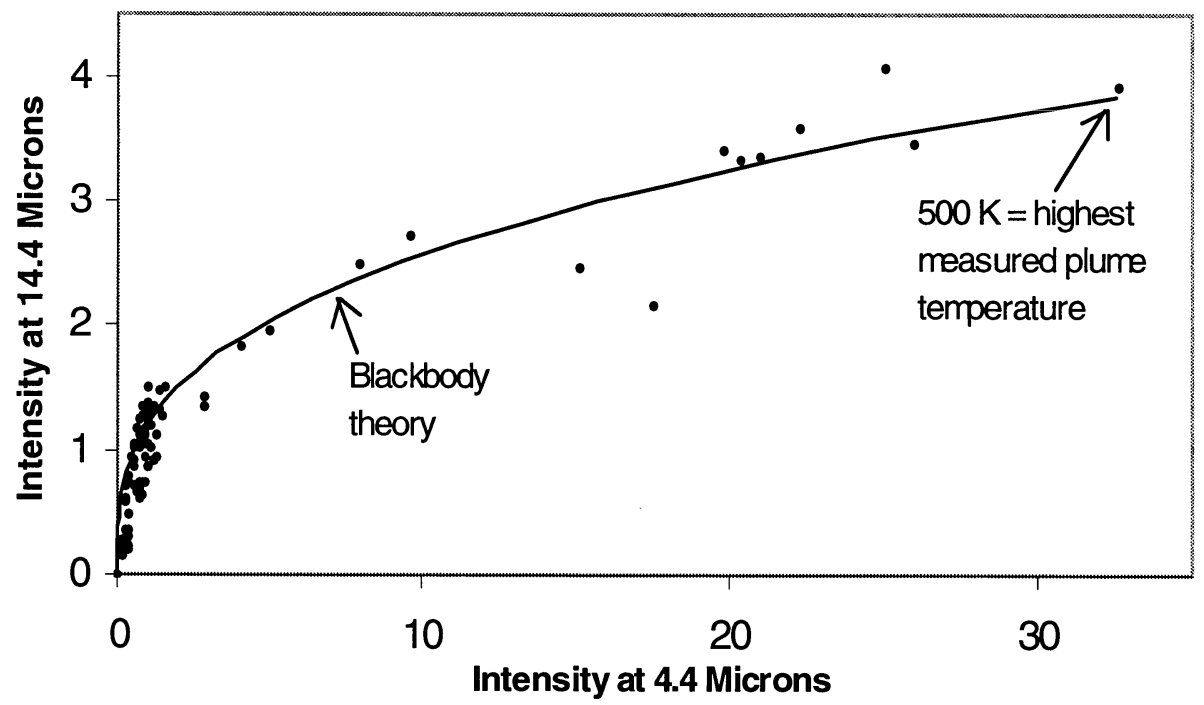

Figure 10. Signal at $14.4371 \mu m$ vs. signal at $4.40462 \mu m$ (Waxahachie data).

The data fit the blackbody prediction quite well. ${ }^{*}$ This does not mean that the plume is literally a blackbody (optically thick), only that blackbody intensity ratios fit the data about as well as a model derived from the Boltzmann distribution would do. Since Waxahachie thermocouple data are not available at this time, the temperatures corresponding to the various data points are not known. However, by correlating the data points with the locations of the spectrometer, it is found that the points far from the origin correspond to observations close to the stack. These are therefore hightemperature points, consistent with their placement on the curve. The data strongly suggest that the ratio of the received signal at these two wavelengths correlates with temperature, in a manner consistent with the blackbody equation.

A similar analysis was done with the vehicle exhaust plume data. The wavelengths used were $4.20772 \mu \mathrm{m}$ (to avoid optical thickness) and $15.3328 \mu \mathrm{m}$ (to avoid interference from analyte spectra). Results are shown in Figure 11.

*In Figure 10, the point that deviates the most from the theoretical curve, with $\mathrm{x}$-coordinate $=17.6$, was taken under identical conditions as, and within one minute of, the well-behaved point with $\mathrm{x}$-coordinate $=26$. The plume may have drifted away from the line of sight during part of this measurement, causing the spectrum to be reduced uniformly, at all wavelengths. This interpretation is supported by noting that these two points are nearly collinear with the origin. 


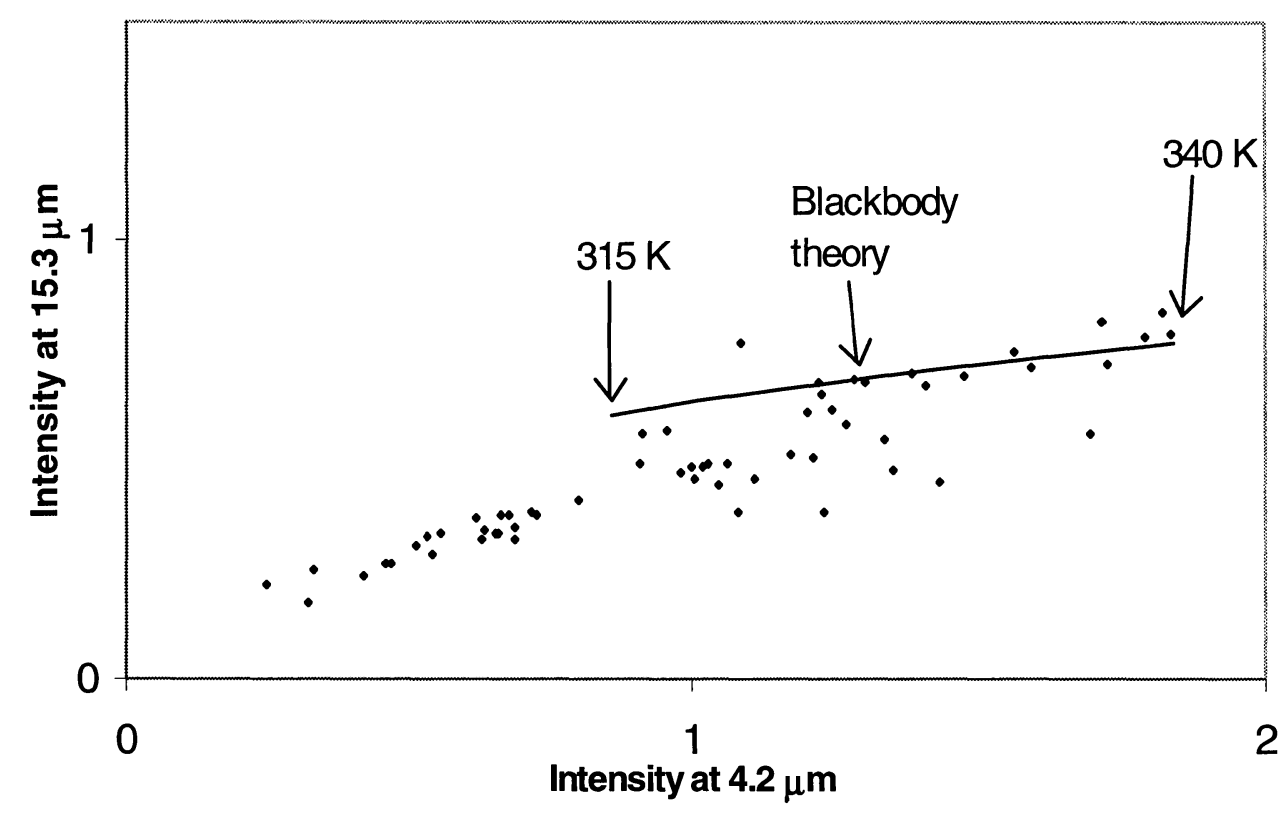

Figure 11. Signal at $15.3328 \mu \mathrm{m}$ vs. signal at $4.20772 \mu \mathrm{m}$ (vehicle exhaust plume data).

The vehicle exhaust plume data show much more scatter than the Waxahachie data. The density of the plume was probably variable (since the plume is optically thin at these two wavelengths, the signals depend on concentration). Such fluctuations in $\mathrm{CO}_{2}$ concentration are to be expected, since a considerable amount of air was injected into the plume along with the analytes. A blackbody prediction, scaled to the data, is also shown in Figure 11. Some of the data are associated with thermocouple measurements; points on the theoretical curve corresponding to the minimum and maximum measured temperatures ( 315 and $340 \mathrm{~K}$ ) are indicated. Clearly, the temperature range is too small to show a parabolic curve, as was seen in Figure 10. The data show a much larger intensity variation than the temperatures would lead one to expect (i.e., there are many points to the left of the theoretical minimum). This variation supports the conclusion that the vehicle exhaust plume had large density fluctuations.

Since temperatures are available for the vehicle exhaust plume, both from thermocouple measurements and from the intensity of the optically thick transition (Section 4.2.1), it is possible to investigate directly the dependence of the intensity ratio on temperature. Results are shown in Figure 12, along with a scaled theoretical curve derived from the blackbody equation. Circles indicate data points with associated thermocouple measurements; crosses indicate temperatures inferred from the intensity of the optically thick transition at $4.25246 \mu \mathrm{m}$. The data appear consistent with theory. Note that the ratio plotted on the ordinate and the optically thick emissions used to calculate the temperatures on the abscissa are both insensitive to density fluctuations. Hence, it is not surprising that the points in Figure 12 are better behaved than those in Figure 11. The preliminary conclusion is that the ratio of signals at widely-separated wavelengths scales with temperature in a predictable and useful manner. The points show some scatter, however, and data that span a wider temperature range are needed to verify this conclusion.

\section{CONCLUSIONS}

In the work reported in this paper, spectroscopic measurements were combined with thermocouple "ground-truthing" to examine plume physics in detail. Two spectroscopic temperature diagnostics were found to correlate well with thermocouple measurements. If an optically thick transition is available, its intensity can be used with the Planck equation to provide a temperature estimate. In the more common case of optically thin transitions, the ratio between spectral intensities at widely-separated wavelengths can be used as a plume temperature diagnostic. Since the ratio of two optically thin measurements is independent of gas concentration, density fluctuations in the plume are normalized out by this technique. Fine spectral resolution is not required. 


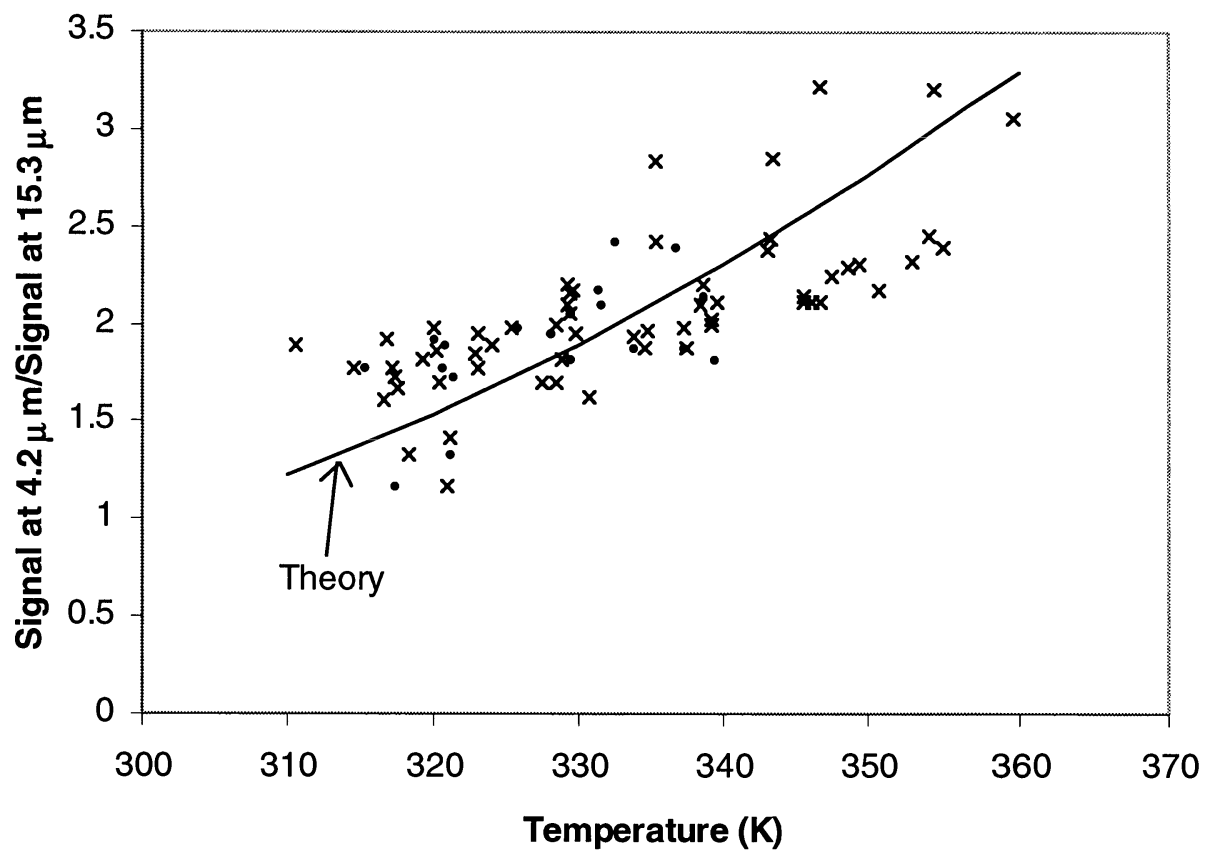

Figure 12. Ratio of signal at two wavelengths vs. temperature (vehicle exhaust plume).

The ratio between the intensities of two molecular transitions should be proportional to the Boltzmann ratio

$$
R_{\text {Boltzmann }}=\frac{\exp \left(c h / \lambda_{1} k T\right)}{\exp \left(c h / \lambda_{2} k T\right)}
$$

where $\lambda_{1}$ and $\lambda_{2}$ are the wavelengths of the two transitions. For most plumes, this factor differs little from the ratio derived from the blackbody Planck equation

$$
R_{\text {Planck }}=\frac{\exp \left(c h / \lambda_{1} k T\right)-1}{\exp \left(c h / \lambda_{2} k T\right)-1}
$$

The results of this research suggest that it makes little difference which of these ratios is used.

It should be emphasized that optically thin plumes are not blackbodies. The magnitudes of signals at different wavelengths, taken individually, are not predictable from blackbody theory unless they are optically thick. The plume data presented in this report are proportional to the ratios above, but the ratios must be calibrated by appropriate multiplying factors to fit the data. This procedure is a correction for the intrinsic strengths of the molecular transitions, which will generally differ from the blackbody curve. If the ratio is known at some particular temperature, Equation (7) or Equation (8) can be used to predict the ratio at other temperatures. Comparison with the measured ratio provides the temperature diagnostic. If both transitions derive from the same molecule and are spectrally isolated, the calibration factor may be independent of plume composition, and the calibration factor derived from one plume may apply to other plumes. If two molecular species are involved (at unknown relative concentrations), the calibration factor will be plumespecific. A large amount of plume data must then be taken and fit to an adjusted theoretical curve, as was done in this research. Once the calibration is done, it should remain valid unless the composition of the plume changes.

Additional work is needed to assess the potential of this technique. Thermocouple measurements that span a range of more than $100 \mathrm{~K}$ were taken during the Waxahachie collection; comparison of these data with temperatures derived from the spectroscopic ratio technique will provide a stringent test. Work with additional spectral transitions is also needed, since the $\mathrm{CO}_{2}$ lines used in this study are convenient, but may be problematical for high-altitude measurements due to atmospheric absorption. To validate the technique for practical use, it should be applied to radiation from molecules not present in the atmosphere. Usable molecular transitions from various plume species need to be identified, and their 
calibration factors determined. The accuracy and reproducibility of the measurements need to be studied and related to the amount of turbulence present in the plume. Finally, the time response and spatial resolution of the spectral measurements should be assessed. If the ongoing and planned research confirms the preliminary findings reported in this paper, a simple, practical, and robust plume temperature diagnostic may be available to the remote sensing community.

\section{ACKNOWLEDGEMENTS}

The Waxahachie collection was a collaborative effort between the Central MASINT Organization Technology Coordination Office, SITAC, Los Alamos National Laboratory, and the Environmental Protection Agency (EPA) Region-7, and was conducted under the auspices of the EPA. Critical assistance at Waxahachie was provided by experiment design leads Robert Kroutil of Los Alamos National Laboratory and Mark Thomas of the Environmental Protection Agency, as well as participants from AeroSurvey, Airborne Imaging, and McKinzie Environmental. SITAC's efforts also benefited from the contributions of experimenters from ABB Bomem and Rochester Institute of Technology. Craig Miller and Joe Leckie provided able assistance with the experimental setup and measurements. The authors also thank Christine Kervina for technical editing assistance.

\section{BIBLIOGRAPHY}

1. Levine, I. N., Molecular Spectroscopy, Wiley-Interscience, New York (1975), p. 259.

2. Chan, S. H., Lin, C. C., and Low, M. J. D., "Analysis of Principles of Remote Sensing and Characterization of Stack Gases by Infrared Spectroscopy," Environ. Sci. Technol., vol. 7, pp. 424-427 (1973).

3. Herget, W. F. and Brasher, J. D., "Remote Fourier Transform Infrared Air Pollution Studies," Opt. Eng., vol. 19, pp. 508-514 (1980).

4. Herget, W. F., "Remote and Cross-Stack Measurement of Stack Gas Concentrations Using a Mobile FT-IR System," Appl. Opt., vol. 21, pp. 635-641 (1982).

5. Gross, L. A., Griffiths, P. R., and Sun, J. N.-P., "Temperature Measurement by Infrared Spectroscopy," in Infrared Methods for Gaseous Measurements, J. Wormhoudt, editor, Marcel Dekker, Inc., New York, pp. 81-137 (1985).

6. Haus, R., Schäfer, K., Bautzer, W., Heland, J., Mosebach, H., Bittner, H., and Eisenmann, T., "Mobile FourierTransform Infrared Spectroscopy Monitoring of Air Pollution," Appl. Opt., vol. 33, pp. 5682-5689 (1994).

7. Gross, L. A. et al. (1985).

8. Prengle, H. W., Morgan, C. A., Fang, C.-S., Huang, L.-K., Camapani, P., and Wu, W. W., "Infrared Remote Sensing and Determination of Pollutants in Gas Plumes," Environ. Sci. \& Technol., vol. 7, pp. 417-423 (1973).

9. Prengle, H. W., Mahagaokar, U., and Tse, S.-K., "Thermal and Momentum Structure of an Emerging Plume by Remote Sensing," in Infrared Methods for Gaseous Measurements, J. Wormhoudt, editor, Marcel Dekker, Inc., New York, pp. 47-79 (1985).

10. Solomon, P. R., Morrison, P. W., and Serio, M. A., "Fourier Transform Infrared Spectroscopy for Process Monitoring and Control," Industrial, Municipal, and Medical Waste Incineration Diagnostics and Control, SPIE vol. 1717, pp. 104-115 (1992).

11. Young, S. J., "Detection and Quantification of Gases in Industrial-Stack Plumes Using Thermal-Infrared Hyperspectral Imaging,” The Aerospace Corporation, El Segundo, CA, ATR-2002 (8407)-1 (2002), pp. 53-55.

12. Chaffin, C. T. and Marshall, T. L., "Generating Well-Characterized Chemical Plumes for Remote Sensing Research," Electro-Optical Technology for Remote Chemical Detection and Identification III, SPIE vol. 3383, pp. 113-123 (1998).

13. Mitchell, H. J., Jellison, G. P., Miller, D. P., Salvaggio, C., and Miller, C., "The Importance of Background in the Detection and Identification of Gas Plumes Using Emissive Infrared Hyperspectral Sensing," Algorithms and Technologies for Multispectral, Hyperspectral, and Ultraspectral Imagery IX, SPIE vol. 5093 (2003).

14. Brown, J. M., Molecular Spectroscopy, Oxford University Press, Oxford (1998), p. 48.

15. Williams, D., "Molecular Spectroscopy: Infrared Region," in Methods of Experimental Physics, Vol. 13: Spectroscopy, D. Williams, editor, Academic, New York, 1976, pp. 36-37.

16. Herzberg, G., Molecular Spectroscopy and Molecular Structure, Vol. I: Spectra of Diatomic Molecules, Van Nostrand, New York (1950), pp. 125-126. 